ARTICLE

https://doi.org/10.1038/s41467-018-08285-5

\title{
Control of structural flexibility of layered-pillared metal-organic frameworks anchored at surfaces
}

\author{
Suttipong Wannapaiboon ${ }^{1,2}$, Andreas Schneemann (1) 1, Inke Hante ${ }^{3}$, Min Tu ${ }^{3,4}$, Konstantin Epp \\ Anna Lisa Semrau (1) ${ }^{1}$, Christian Sternemann ${ }^{5}$, Michael Paulus ${ }^{5}$, Samuel J. Baxter ${ }^{6}$, Gregor Kieslich ${ }^{1} \&$ \\ Roland A. Fischer (iD ${ }^{1}$
}

Flexible metal-organic frameworks (MOFs) are structurally flexible, porous, crystalline solids that show a structural transition in response to a stimulus. If MOF-based solid-state and microelectronic devices are to be capable of leveraging such structural flexibility, then the integration of MOF thin films into a device configuration is crucial. Here we report the targeted and precise anchoring of $\mathrm{Cu}$-based alkylether-functionalised layered-pillared MOF crystallites onto substrates via stepwise liquid-phase epitaxy. The structural transformation during methanol sorption is monitored by in-situ grazing incidence $X$-ray diffraction. Interestingly, spatially-controlled anchoring of the flexible MOFs on the surface induces a distinct structural responsiveness which is different from the bulk powder and can be systematically controlled by varying the crystallite characteristics, for instance dimensions and orientation. This fundamental understanding of thin-film flexibility is of paramount importance for the rational design of MOF-based devices utilising the structural flexibility in specific applications such as selective sensors.

\footnotetext{
${ }^{1}$ Chair of Inorganic and Metal-Organic Chemistry, Department of Chemistry, Technical University of Munich, Lichtenbergstraße 4, 85787 Garching, Germany. ${ }^{2}$ Synchrotron Light Research Institute (Public Organization), 111 University Avenue, Muang District, Nakhon Ratchasima 30000, Thailand. ${ }^{3}$ Chair of Inorganic Chemistry II, Faculty of Chemistry and Biochemistry, Ruhr-University Bochum, Universitätstraße 150, 44801 Bochum, Germany. ${ }^{4}$ Centre for Surface Chemistry and Catalysis, Katholieke Universiteit Leuven, Celestijnenlaan 200fBox 24613001 Leuven, Belgium. ${ }^{5}$ Fakultät Physik/DELTA, Technische Universität Dortmund, Maria-Goeppert-Mayer Straße. 2, 44227 Dortmund, Germany. ${ }^{6}$ School of Chemistry and Biochemistry, Georgia Institute of Technology, Atlanta, GA 30332, USA. Correspondence and requests for materials should be addressed to R.A.F. (email: roland.fischer@tum.de)
} 
$\mathrm{F}$ lexible materials are intriguing for the development of novel composites, due to their responsiveness to specific stimuli by undergoing structural transformation ${ }^{1-5}$. For centuries, the potential utility of biological flexible materials has been perceived. Leonardo da Vinci produced the first hygrometer by employing the expansion/contraction of human hair upon ad- and de-sorption of moisture ${ }^{6}$. More advanced responsiveness has been observed in biomimetic and organic materials such as enzymes, biomolecules and stimuli-responsive polymers. These materials can change their conformation in response to external stimuli and adapt their structures to surrounding environments. Consequently, they can transduce chemical and biological signals into optical, electrical and mechanical signals leading to various applications such as smart optics, biosensors and electromechanical systems ${ }^{5}$. Even though flexibility is quite common in elastic polymers, creating flexible, crystalline solid-state materials remains a challenge, since their long-range compositional ordering may prevent spatial rearrangement. Only few examples of zeolites show some structural adaptivity in response to highpressure compression, however still depending on the hydrostatic pressure-transmitting media ${ }^{7,8}$. However, recently, structural changes in elastic crystals of copper(II) acetylacetonate during mechanical stress have been reported ${ }^{9}$.

Metal-organic frameworks (MOFs) are a promising class of porous, crystalline materials, formed by the combination of metal ions or clusters and multitopic organic linkers, which allows to integrate flexibility and responsivity into rigid, crystalline structures ${ }^{10}$. Due to the versatility of precursor building blocks and the possibility for chemical functionalisation of coordination space, MOFs offer a broad perspective for rationally designing and fine tuning their structure, characteristic features and chemical properties of the pore surface ${ }^{11-14}$. The specific design of MOF components to control attractive forces used for assembling the porous scaffolds can initiate structural dynamics in some MOFs (named flexible MOFs or soft porous crystals (SPCs)) $)^{10,15-18}$. Depending on their characteristic features, SPCs can undergo reversible phase transitions in various flexible modes (i.e., breathing ${ }^{19}$, swelling ${ }^{20}$, ligand rotation ${ }^{21}$, subnetwork displacement ${ }^{22,23}$ ) upon external stimuli such as guest sorption, temperature change, light and mechanical pressure ${ }^{15,16}$. The discovery of highly-responsive MOFs highlights the potential applications for selective gas storage ${ }^{24-26}$, effective gas separation $^{27}$, controlled drug release ${ }^{28}$ and also smart sensors ${ }^{29}$.

The so-called breathing transition in MOFs has been extensively studied for the prototypical class of $\mathrm{M}(\mathrm{bdc})(\mathrm{OH})$ frameworks $\left(\right.$ MIL-53, $\mathrm{MIL}=$ Materiaux Institute Lavoisier; $\mathrm{M}^{3+}=\mathrm{Al}^{3+}, \mathrm{Fe}^{3+}$, $\mathrm{Cr}^{3+}, \mathrm{Sc}^{3+}, \mathrm{Ga}^{3+}$, and $\mathrm{In}^{3+}$; $\mathrm{bdc}^{2-}=1,4$-benzenedicarboxylate). These frameworks are built up by one dimensional $\mathrm{M}-\mathrm{OH}-\mathrm{M}$ chains bridged by bdc $\mathrm{bd}^{2-}$ ligands and typically undergo (in the case of $\left.\mathrm{M}=\mathrm{Al}^{3+}, \mathrm{Cr}^{3+}, \mathrm{Ga}^{3+}\right)$ a large pore (lp) to narrow pore (np) phase transition upon guest adsorption in the activated (i.e., solvent free) state ${ }^{19,30}$. However, the utilisation of different metal centres (i.e., $\left.\mathrm{Fe}^{3+}, \mathrm{Sc}^{3+}\right)^{31,32}$ and $\mathrm{bdc}^{2-}$ derivatives featuring functional groups $^{33}$ modifies the breathing effect substantially. These transitions are usually identified and analysed by X-ray diffraction techniques (in-situ and ex-situ) but are also indicated by steps in the adsorption isotherm ${ }^{16}$. Apart from MIL-53, layered-pillared MOFs $\left(\mathrm{M}_{2} \mathrm{~L}_{2} \mathrm{P} ; \mathrm{M}=\right.$ metal ion centre; $\mathrm{L}=$ linear dicarboxylate linker; $\mathrm{P}=$ neutral nitrogen donor pillar) show under particular circumstances breathing transition. The prototypic $\mathrm{Zn}_{2}$ (bdc) ${ }_{2}($ dabco $)($ dabco $=1,4$-diazabicyclo[2.2.2] octane) consists of a square-grid $\mathrm{Zn}_{2}(\mathrm{bdc})_{2}$ lattices featuring $\mathrm{Zn}_{2}$ paddlewheels, bridged by dabco pillars along the axial position of the paddlewheel. This material reveals small structural transition upon adsorption of certain guest molecules (i.e., DMF and benzene) due to the deformation of the paddle-wheel $\mathrm{Zn}_{2}(\mathrm{bdc})_{2}$ grids when interacting with the guests ${ }^{34}$, however, if isopropanol is adsorbed inside the structure, a considerable breathing transition is observed $^{35}$. Along these lines, functionalisation of the dicarboxylate linkers can introduce and even tailor the substantial responsiveness ${ }^{36,37}$. We illustrated in our previous works that the incorporation of alkylether-functionalised bdc linkers in $\mathrm{Zn}_{2}(\mathrm{fu}-$ bdc) ${ }_{2}$ (dabco) compounds (fu-bdc $=2,5$-alkoxy-functionalised-1,4benzenedicarboxylate) induces a breathing effect upon polar guest sorption $^{37}$ and temperature change ${ }^{38}$. The dynamic breathing phenomenon between the guest-filled lp form and the guest-free np form can be finely tuned by changing the characteristics of the pendant groups ${ }^{37}$, and recently also by the choices of metal nodes $^{39}$. Moreover, mixing of different metal nodes or different linkers is another strategy to tune the structural responsiveness ${ }^{40}$.

In addition to modifying the MOF composition, controlling their mesoscopic physical form such as crystal size and morphology is known to uniquely affect their properties ${ }^{41}$. Downsizing of flexible MOF crystallites to the nanoscale regime can alter their adsorption profiles and even generate new intrinsic features differing from the bulk $\mathrm{MOFs}^{42-45}$. For example, the bulk form of the $\mathrm{Cu}_{2}(\mathrm{bdc})_{2}$ (bipy) compound (bipy $=4,4^{\prime}$-bipyridine) shows an opened-pore (op) to closed-pore (cp) structural transition upon guest desorption, while the nanocrystalline form $(<60 \mathrm{~nm})$ remains in the op after guest removal. Transformation to the $\mathbf{c p}$ form is only achieved by thermal treatment ${ }^{43}$. Moreover, DUT-8(Ni) $\left(\mathrm{Ni}_{2}(\mathrm{ndc})_{2}(\mathrm{dabco}) ; \mathrm{ndc}^{2-}=2,6\right.$-naphthalenedicarboxylate) also exhibits size-dependent framework flexibility upon guest sorption ${ }^{44}$. Small DUT-8(Ni) crystals $(<500 \mathrm{~nm})$ retain the op after activation, whereas larger DUT-8(Ni) crystals $(>1 \mu \mathrm{m})$ undergo the op-to-cp breathing transition after thermal activation $^{44}$

The implementation of MOFs into thin films has received increasing attention over the last few years ${ }^{46,47}$, since it is imperative for the advancement of MOF-based devices. Key challenges in their fabrication are the ability to engineer and enhance characteristic MOF features at the nanoscale, and to spatially control crystallisation processes to be compatible with device configurations ${ }^{46-48}$. Among various methods, stepwise liquid-phase epitaxy (LPE) is beneficial for the formation of homogeneous MOF thin films with controllable thickness. Moreover, the crystallite orientation can be controlled within the LPE process by varying the surface functionality (e.g., by using self-assembled monolayers (SAM) of organothiols featuring different terminal-groups ${ }^{49}$. This method is driven by the alternating immersion of a substrate into solutions containing individual MOF precursor components ${ }^{49,50}$. Even though various MOFs with rigid structures have been fabricated as thin films, studies on flexible MOF thin films remain scarce. The first attempt was the preparation of a $\mathrm{Fe}_{3} \mathrm{O}\left(\mathrm{H}_{2} \mathrm{O}\right)_{2} \mathrm{X}(\mathrm{bdc})_{3}\left(\mathrm{X}=\mathrm{F}^{-}\right.$or $\mathrm{OH}^{-}$; MIL-88B (Fe)) film by solvothermal synthesis on a SAM-functionalised Aucoated substrate. This micrometre-sized crystalline film exhibits framework swelling induced by moisture adsorption in a similar manner to the bulk MIL-88B crystal $^{51}$. Kitagawa and co-workers employed the LPE method to fabricate nanometre-thick interdigitated Hofmann-type MOF thin films, which compellingly exhibit unique structural motifs which influence the adsorption profiles as compared to the bulk MOFs ${ }^{52,53}$. Remarkably, downsizing of the non-porous $\mathrm{Fe}(\mathrm{py})_{2}\left[\mathrm{Pt}(\mathrm{CN})_{4}\right]$ ( $\mathrm{py}=$ pyridine) to a thin film of 16 nanometres thickness initiates a gate-opening structural transformation (cp-to-op) induced by the lower potential energy barrier ${ }^{53}$. At the nanoscale, guests can diffuse in between neighbouring interdigitated layers and move them apart. Increasing the film thickness above a certain threshold leads to a suppression of the flexibility as observed for the bulk material ${ }^{53}$.

In depth understanding of the structural responsiveness and other emerging effects when anchoring flexible MOF crystallites 
onto substrates is necessary to facilitate the development of flexible MOF-based devices such as highly-selective sensors. Herein, we describe the fabrication of alkylether-functionalised $\mathrm{Cu}_{2}(\mathrm{DE}-\mathrm{bdc})_{2}(\mathrm{dabco}) \quad(\mathbf{1})$ and its analogue $\mathrm{Cu}_{2}(\mathrm{BME}-$ bdc) ${ }_{2}$ (dabco) (2) as thin films (DE-bdc $=2,5$-diethoxy-1,4-benzenedicarboxylate and $\mathrm{BME}-\mathrm{bdc}=2,5$-bis(2-methoxyethoxy) -1,4-benzenedicarboxylate) on SAM-functionalised Au-coated quartz crystal microbalance (QCM) sensors. The growth behaviours and the adsorption profiles are investigated by tracking the QCM frequency change, which is proportional to the mass change based on the Sauerbrey Equation. A study of guestinduced structural flexibility of the thin-films in comparison with the bulk MOFs is performed by in-situ synchrotron grazing incidence X-ray diffraction (GIXRD) during guest adsorption by a custom-built, semi-quantitative vapour adsorption unit (Supplementary Fig. 4). Notably, unique and different structural responsiveness upon methanol sorption in the thin films is observed. This highlights not only the striking effect of anchoring the flexible MOF crystallites onto the substrate surface, but also the marked dependence on the number of LPE deposition cycles (i.e., film thickness) (Fig. 1).

\section{Results}

Structural flexibility of bulk powders. Polycrystalline powder of 1 (1bulk) was synthesised and characterised to establish a benchmark data set for comparison of the structural flexibility of $\mathbf{1}$ in bulk and as a thin film. 1bulk was prepared by solvothermal reaction of $\mathrm{Cu}\left(\mathrm{NO}_{3}\right)_{2} \cdot 3 \mathrm{H}_{2} \mathrm{O}$ with $\mathrm{H}_{2}$ DE-bdc and dabco (Fig. 2a) in $\mathrm{DMF}$ at $120^{\circ} \mathrm{C}$ for $48 \mathrm{~h}$. Upon activation in vacuo overnight at $130{ }^{\circ} \mathrm{C}$, 1bulk undergoes a structural transformation from the solvated, $\mathbf{l p}$ form to the guest-free, $\mathbf{n p}$ form. After solvent removal from the pore of 1bulk, the neighbouring alkyletherfunctionalised sidechains can interact with each other. Referring to the literatures ${ }^{37,39}$, single crystal XRD was used to identify the structural evidence of the as-synthesised (solvated) form of $\mathrm{Zn}$ based alkoxyether-functionalised layered-pillared MOFs and Pawley refinements were used to identify the change of lattice parameters with respect to the breathing phenomena upon guest adsorption and desorption. This refinement indicated the wine rack-like motion which changes the square $\mathrm{M}_{2}$ (fu-bdc) grids in the solvated, $\mathbf{l p}$ form into rhombic grids in the activated, np form, while the dabco-containing axis remains mostly constant (Supplementary Fig. 5). Herein, we performed Pawley refinements of the lattice parameters of the $\mathrm{MeOH}$-solvated and activated forms of $\mathbf{1}$ bulk and $\mathbf{2 b u l k}$ from their GIXRD patterns (using synchrotron X-ray radiation with a wavelength of $0.827 \AA$ ). (Supplementary Figs. 5 and 7, Supplementary Table 1). In good agreement with the literature, we observe from the Pawley refinements that $\mathbf{1 b u l k}$ (as well as 2bulk) shows an increase of lattice parameter $a$ and a decrease of lattice parameter $b$, while lattice parameter $c$ remains constant upon $\mathrm{MeOH}$ desolvation. Consequently, these changes of the unit cell parameters lead to a reduction of the total unit cell volume, indicating the guest induced breathing transition (from the solvated, lp form to the activated, np form $)^{37,39}$. These evidences confirm the structural transition of the copper-based materials 1bulk and 2bulk in a similar manner to their zinc analogues, as well as their thin-films during the sorption of methanol.

We further investigated the structural flexibility of $\mathbf{1 b u l k}$ as a response to polar-guest sorption (herein, methanol vapour) at ambient temperature by in-situ GIXRD. According to the GIXRD patterns (Fig. 2b), adsorption of methanol at relative vapour pressure $\left(P / P_{0}\right)$ of $10 \%$ in a dynamic He stream (blue line plots of GIXRD patterns) initiates the breathing transition in 1bulk from the activated, $\mathbf{n p}$ form (the main diffraction peak is highlighted with a yellow bar) to the solvated, lp form (highlighted with a green bar). A complete transformation towards the lp form occurs after the relative vapour pressure of the methanol stream is increased to $20 \% \mathrm{P} / \mathrm{P}_{0}$. Purging the $\mathrm{MeOH}$-solvated 1bulk with dry $\mathrm{He}$ gas at $25^{\circ} \mathrm{C}$ desorbs methanol and the initial np phase is regained (wine-colour line plots of GIXRD patterns). In addition, polycrystalline powder of $\mathbf{2}$ (2bulk) exhibits a similar trend of the structural flexibility upon methanol sorption at $25^{\circ} \mathrm{C}$, even though the change of the refined lattice parameters is different (Supplementary Fig. 10). This observation illustrates a way to modulate the structural responsiveness in MOFs even at room temperature by varying size and functionality of the pendent sidechains, which can interact with the guest molecules ${ }^{37}$.

Fabrication of MOF thin-films and their crystallinity. Aiming for high-performance MOF-based devices, we herein focus on the implementation of structural flexibility into MOF thin films onto QCM sensors. A stepwise LPE process is used for the fabrication of 1 and 2 thin films with varying deposition cycles, hereafter named as $\mathbf{1} \mathbf{t f} \mathbf{x}_{\mathbf{x}}$ and $\mathbf{2} \mathbf{t f} \mathbf{f}_{\mathbf{x}}$ ( $x=$ number of deposition cycles). In the LPE process, solutions of $\mathrm{Cu}(\mathrm{OAc})_{2}$ and the mixed organic linkers are alternatingly dosed to the $-\mathrm{COOH}$ terminated SAMfunctionalised QCM substrate in a continuous flow reactor for 40, 60,80 and 120 cycles. Importantly, each precursor dosing step is followed by flushing of the reactor cell with ethanol. The applied LPE procedure is schematically shown in Fig. 3a. The deposition onto a QCM substrate allows for measurements of the change of QCM oscillator frequency and in turn in-situ investigation of the film growth is possible. We observe an effective control of the deposited sample mass on the substrate as a function of deposition cycles, which implies stepwise construction of the MOF crystallites (Fig. 3b). A closer inspection of the QCM frequency change confirms saturation during each deposition step of the metal and the mixed organic linkers, which reflect a selfterminated growth of the layered-pillared MOF thin films (Supplementary Fig. 11).

Considering the LPE growth of $\mathbf{1 t f}_{\mathbf{x}}$ on the $-\mathrm{COOH}$ terminated QCM substrates, the as-synthesised thin films exhibit a high crystallinity with a preferred orientation along the (110) plane of the solvated, lp form (black line plots of the out-of-plane GIXRD patterns illustrated in Fig. 4), referring to the $\mathrm{Zn}$-based analogous structure reported in the literature ${ }^{37}$ and the refined lattice parameters of 1 bulk in Supplementary Fig. 6. According to these results, the $\mathrm{Cu}_{2}(\mathrm{DE}-\mathrm{bdc})_{2}$ grids in $\mathbf{1 t f}_{\mathbf{x}}$, which can exhibit a wine rack-like motion during the breathing phenomenon, are oriented orthogonally to the substrate surface. Moreover, crystallite sizes were calculated using the Scherrer equation from the GIXRD patterns of $\mathrm{MeOH}$-solvated $\mathbf{1 t f}_{\mathbf{x}}$ samples (see Supplementary Fig. 12). There is no significant difference of the calculated crystallite size of $\mathbf{1} \mathbf{t f}_{\mathbf{x}}$ samples. Note that, Scherrer equation is not applicable for particles with a size larger than about 100 or 200 $\mathrm{nm}$. Considering the particle size within the $\mathbf{1 t f}_{\mathbf{x}}$ samples (see SEM images discussed in detail in the following section), the observed particle sizes range within the sub-micrometres scale. Hence, the calculated crystallite sizes of $\mathbf{1 t f}_{\mathbf{x}}$ based on the Scherrer equation could be highly deviated from the actual size. Therefore, they cannot be properly used to identify the size difference of the obtained $\mathbf{1 t f}_{\mathbf{x}}$ samples and consequently the effect on structural flexibility of the samples reported herein.

Structural flexibility upon methanol sorption of MOF thinfilms. In order to examine the structural responsiveness of $\mathbf{1 t f}_{\mathbf{x}}$ in comparison to lbulk and understand the influence of the crystallite dimension on the framework flexibility, GIXRD patterns are 
1bulk
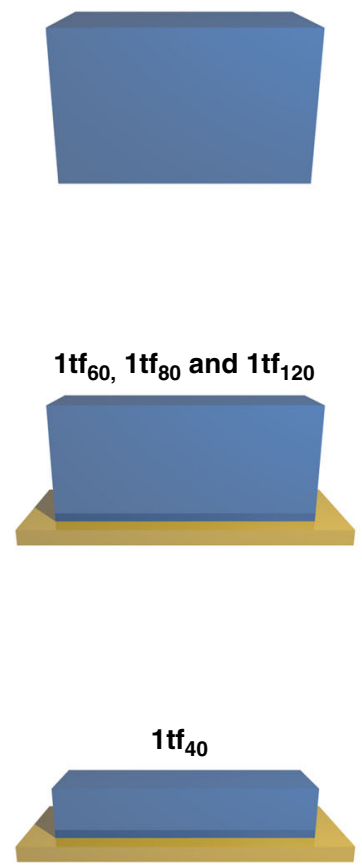
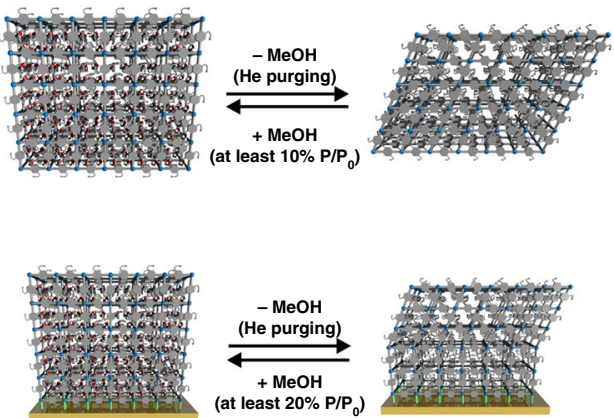

(at least $20 \% \mathrm{P}$

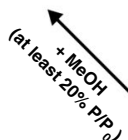

$\left.P / P_{0}\right)$
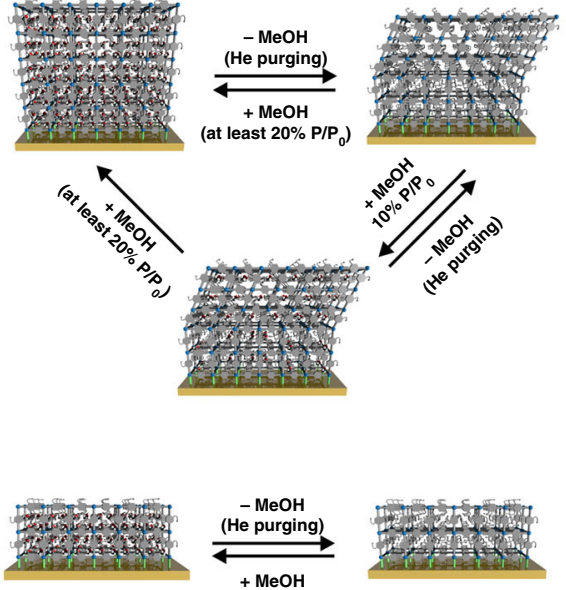

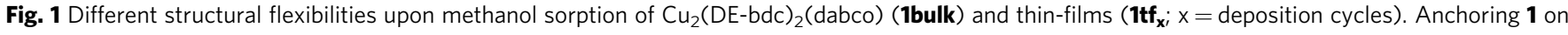
the surface affects the structural responsiveness induced by guest sorption, which shows unique breathing behaviour in dependence of the total number of LPE deposition cycles (in other words, the crystallite dimension). Note that, the schematic structures are only suggested structures based on unit cell refinements (Pawley method) of GIXRD patterns of the materials and not a depiction of the actual structures

a

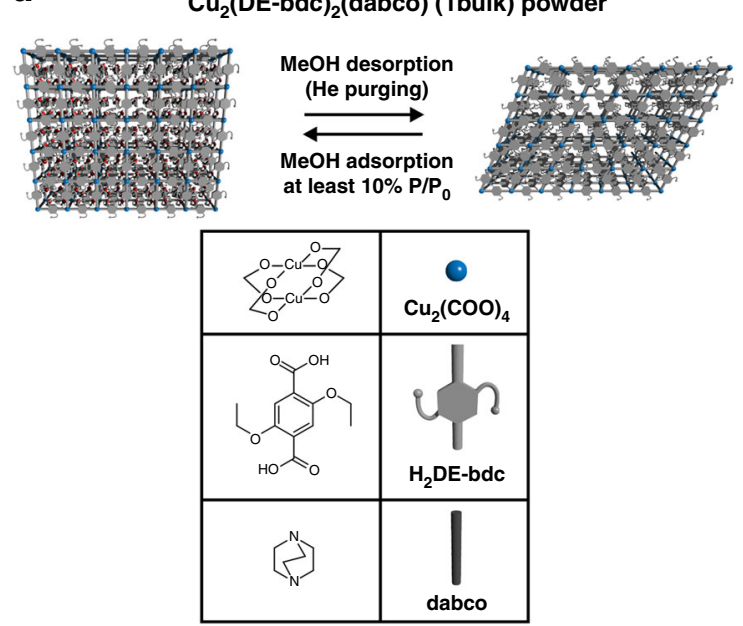

b

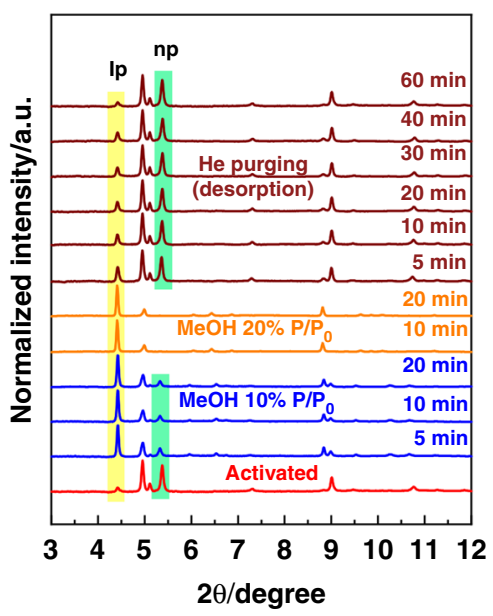

Fig. $\mathbf{2}$ Structural responsiveness of $\mathbf{1 b u l k}$ upon methanol sorption. a Schematic representation of the breathing transformation of $\mathbf{1 b u l k}$ upon methanol sorption and the representations of its structural components; $\mathbf{b}$ In-situ monitoring of GIXRD patterns (X-ray wavelength of $0.827 \AA$ ) during methanol sorption at $25^{\circ} \mathrm{C}$. The structural change from the activated, $\mathbf{n p}$ form to the solvated, Ip form is initiated by adsorption of $10 \% P / P_{0}$ methanol and is complete after adsorption of at least $20 \% \mathrm{P} / \mathrm{P}_{\mathrm{O}}$. Desorption of methanol by purging with dry He gas flow regains the activated, $\mathbf{n p}$ form, which indicates the reversible structural responsiveness of $\mathbf{1 b u l k}$

recorded during dynamic methanol adsorption at $25^{\circ} \mathrm{C}$. Herein, the structural response of $\mathbf{1 t f}_{\mathbf{x}}$ are studied during 4 different stages of the methanol sorption process: (1) the as-synthesised, solvated stage, (2) the activation procedure by purging with dry $\mathrm{He}$ gas (transformation from solvated to guest-free (activated) state), (3) the methanol adsorption at various $P / P_{0}$ (incremental transformation from guest-free to solvated state), and (4) the desorption process (solvated back to guest-free state). The responsiveness of $\mathbf{1 t f}_{\mathbf{x}}$ crystallites is investigated using out-of-plane and in-plane GIXRD profiles.

The out-of-plane GIXRD profiles of $\mathbf{1 t f}_{\mathbf{x}}$ (on $-\mathrm{COOH}$ functionalised QCM substrates, Fig. 4) show a significantly different structural responsiveness upon methanol sorption compared to lbulk. Moreover, we observe a dependency of the structural flexibility on the total number of LPE fabrication cycles. The crystallites in $\mathbf{1 t f}_{\mathbf{4 0}}$ remain in the $\mathbf{l p}$ form regardless of the 
a

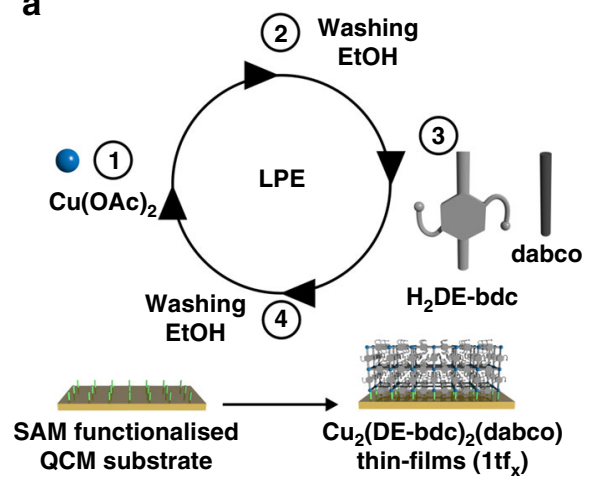

b

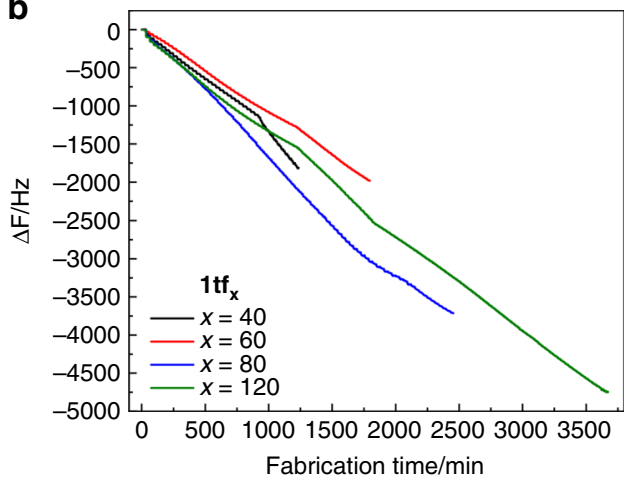

Fig. $3 \mathrm{LPE}$ growth of $\mathbf{1} \mathbf{t f}_{\mathbf{x}}$ and changes in the QCM oscillator frequency $(F)$ as a function of time during the growth. a $\mathbf{1 t f _ { \mathbf { x } }}$ were fabricated via a stepwise $\mathrm{LPE}$ process in the continuous flow mode at $40^{\circ} \mathrm{C}$ for 40 (black plot), 60 (red plot), 80 (blue plot) and 120 cycles (green plot). In each cycle, the QCM substrate was alternatingly exposed to the solutions of metal and mixed organic precursors followed by washing with ethanol after each precursor dosing step; $\mathbf{b}$ In-situ monitoring of the QCM frequency change indicates overall linear-growth profiles with respect to the number of repeated deposition cycles. Increasing the total number of deposition cycles shows a decrease of frequency. According to the Sauerbrey Equation, this observation indicates an increase of mass (i.e., MOF) anchored to the QCM substrate (corresponding to a thicker film with larger crystallite dimension)

treatments (Fig. 4a and Fig. 5a). We attribute this to the strong chemical binding of the MOF crystallites and the SAMfunctionalised surface. This strong surface interaction within $\mathbf{1 t f}_{\mathbf{4 0}}$ presumably inhibits the lp-to-np transformation, either because the strain caused by the structural transformation at the interface cannot be compensated by the beneficial side chain-side chain interactions of the $\mathbf{n p}$ form or it is a size effect as observed in the work of Kitagawa and co-workers on $\mathrm{Cu}_{2}(\mathrm{bdc})_{2}(\text { bipy })^{43}$.

Contrary, $\mathbf{1 t f} \mathbf{f o}_{\mathbf{6 0}}, \mathbf{1 t f}_{\mathbf{8 0}}$ and $\mathbf{1 t f}_{\mathbf{1 2 0}}$ exhibit a structural transformation upon methanol sorption demonstrated by a marked change of the GIXRD patterns. Specifically, the films change from the solvated, $\mathbf{l p}$ form to a mixture of both $\mathbf{l p}$ and $\mathbf{n p}$ forms after activation by purging the samples with $\mathrm{He}$ gas (red plots, Figs. 4b-d). Upon activation, on one hand, the crystallite domains anchored closely to the substrate surface still remain in the guestfree, $\mathbf{l p}$ form due to the restricted framework flexibility as observed for $\mathbf{1 t f}_{\mathbf{4 0}}$. On the other hand, the crystallite domains at a higher distance to the surface, which have less interaction to the substrate, can undergo a breathing transformation and then change from the solvated, $\mathbf{l p}$ form to the guest-free, $\mathbf{n p}$ form (Fig. 5b). Notably, adsorption of $10 \% P / P_{0}$ methanol into the larger-dimension $\mathbf{1 t f}$ films cannot initiate the framework transition towards the solvated, lp form as observed for 1bulk (blue plots, Figs. $4 \mathrm{~b}-\mathrm{d}$ ). Methanol with a $P / P_{0}$ of at least $20 \%$ (orange, green and purple plots, Figs. $4 \mathrm{~b}-\mathrm{d}$ ) is required to trigger the structural flexibility, highlighting the unique effect of anchoring flexible MOFs onto a surface (Fig. 5b). These observations emphasise that the observed $\mathbf{l p}$ and $\mathbf{n p}$ forms in activated $\mathbf{1 t f} \mathbf{f o}_{\mathbf{6 0}}, \mathbf{1} \mathbf{t f}_{\mathbf{8 0}}$ and $\mathbf{1 t f}_{\mathbf{1 2 0}}$ are not a mixture of two different forms of the activated, np 1bulk deposited on top of the activated lp 1tf (see Supplementary Fig. 13). The framework flexibility in $\mathbf{1 t f}$ is reversible by purging with $\mathrm{He}$, showing the transformation back to the activated stage consisting of both $\mathbf{l p}$ and $\mathbf{n p}$ forms.

Top-view scanning electron microscopy (SEM) images (Supplementary Figs. 15 and 16) show the cubic MOF crystal particles within the $\mathbf{1} \mathbf{t} \mathbf{f}_{\mathbf{x}}$ samples (in the lateral dimensions in parallel to the substrate). Analysis of crystal particle size distribution (Supplementary Fig. 17) indicates that $\mathbf{1 t f}_{\mathbf{4 0}}$ has significantly smaller particle size $(80 \%$ of particle size distribution ranging within $100-300 \mathrm{~nm}$ with an average size of about $200 \mathrm{~nm}$ ) than $\mathbf{1 t f}_{\mathbf{6 0}}$, $\mathbf{1} \mathbf{t f}_{\mathbf{8 0}}$ and $\mathbf{1} \mathbf{t f}_{\mathbf{1 2 0}}$ (80\% of particle size distribution ranging within 250-650 nm with an average size of about $400 \mathrm{~nm}$ ). Moreover, cross-sectional SEM images (Supplementary Fig. 18) also reveal a significant difference of the film thickness of $\mathbf{1 t f}_{\mathbf{4 0}}(430 \mathrm{~nm})$ from $\mathbf{1} \mathbf{t f}_{\mathbf{6 0}}(740 \mathrm{~nm}), \mathbf{1 t f}_{\mathbf{8 0}}(840 \mathrm{~nm})$ and $\mathbf{1 t f}_{\mathbf{1 2 0}}(1000 \mathrm{~nm})$. On the same area of QCM sensors, anchoring of the smaller crystal particles on the substrate $\left(\mathbf{1 t f}_{\mathbf{4 0}}\right)$ may show a significant influence of the surface interaction over the whole particles. This consequence leads to a higher activation barrier for the framework breathing in $\mathbf{1 t f}_{\mathbf{4 0}}$ and hence all the crystallite domains remain in the lp form even in the activated, guest-free condition. Unlike $\mathbf{1 t f}_{\mathbf{4 0}}$, the crystallite domains which are less affected by the surface interaction within the thicker $\mathbf{1 t f}_{\mathbf{6 0}}, \mathbf{1} \mathbf{t f}_{\mathbf{8 0}}$ and $\mathbf{1} \mathbf{t f}_{\mathbf{1 2 0}}$ films consisting of larger particles can undergo the guest-induced framework transition.

Additionally, methanol adsorption isotherms measured at $25^{\circ} \mathrm{C}$ on an environmentally controlled QCM indicate different adsorption profiles within the $\mathbf{1 t f}_{\mathbf{x}}$ series (Fig. 6). A marked difference of the isotherm shape can clearly be identified. Specifically, $\mathbf{1 t f}_{\mathbf{4 0}}$ exhibits a single-step methanol adsorption signalling the presence of only the $\mathbf{l p}$ form after activation due to a restriction of the framework flexibility. In contrast, $\mathbf{1 t f} \mathbf{f o}_{\mathbf{6 0}}, \mathbf{1 t f}_{\mathbf{8 0}}$ and $\mathbf{1} \mathbf{t f}_{\mathbf{1 2 0}}$ show two-step adsorption profiles, which are typically indicative of a guest-induced phase transition. The first step $\left(P / P_{0}\right.$ from 0 to 0.15$)$ is attributed to the adsorption of methanol into both the existing $\mathbf{l p}$ and $\mathbf{n p}$ forms of the activated samples, without inducing the framework transformation. A rapid increase of the adsorbed methanol amount at $P / P_{0}$ of 0.15 indicates the threshold pressure that initiates the np-to-lp frameworks transition, leading to a further increase of the amount adsorbed. At high $P / P_{0}$, all the $\mathbf{1 t f}_{\mathbf{x}}$ samples show nearly-identical adsorption values (of approximately $7.5 \mathrm{mmol}$ of methanol per gram of MOF), since all the crystallites are solely presented in the lp form. This observation also reveals the similar accessibility of the pores of the fabricated $\mathbf{1} \mathbf{t f}_{\mathbf{x}}$ samples, which is independent of the thickness (crystallite dimension) of the thin-films. Note that, all the $\mathbf{1 t f}_{\mathbf{x}}$ samples rather show a single-step desorption profile (Supplementary Fig. 19).

For additional investigations, we fabricated 1tf on pyridylterminated SAM-functionalised QCM substrates by employing LPE deposition for 60 cycles (named as $\mathbf{1 t f}_{\mathbf{6 0 - P y}}$ ). $\mathbf{1 t f}_{\mathbf{6 0 - P y}}$ exhibits preferred growth along the (001)-plane of the framework, along the dabco ligand ${ }^{37}$. Unlike $\mathbf{1 t f}_{\mathbf{6 0}}$, GIXRD profiles during methanol sorption of $\mathbf{1 t f}_{\mathbf{6 0}-\mathbf{P y}}$ (Supplementary Fig. 20) present only the lp form in all sorption treatments. Moreover, $\mathbf{1 t f}_{\mathbf{6 0 - P y}}$ exhibits a single-step methanol adsorption isotherm (Supplementary Fig. 21), with a small deviation from the typical type-I adsorption isotherm at $P / P_{0}$ of 0.15 . This implies the possibility of partial 
a

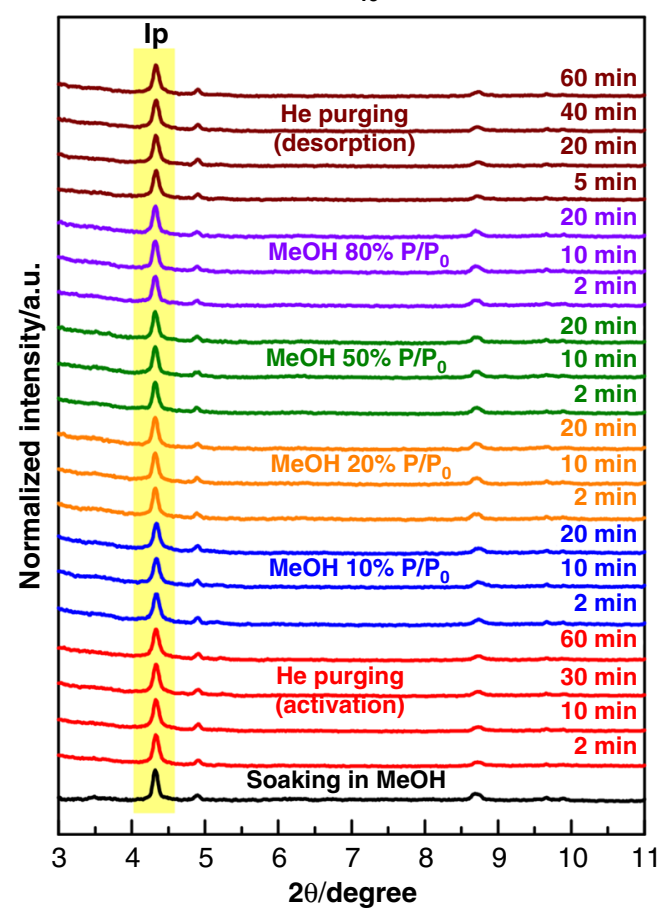

C

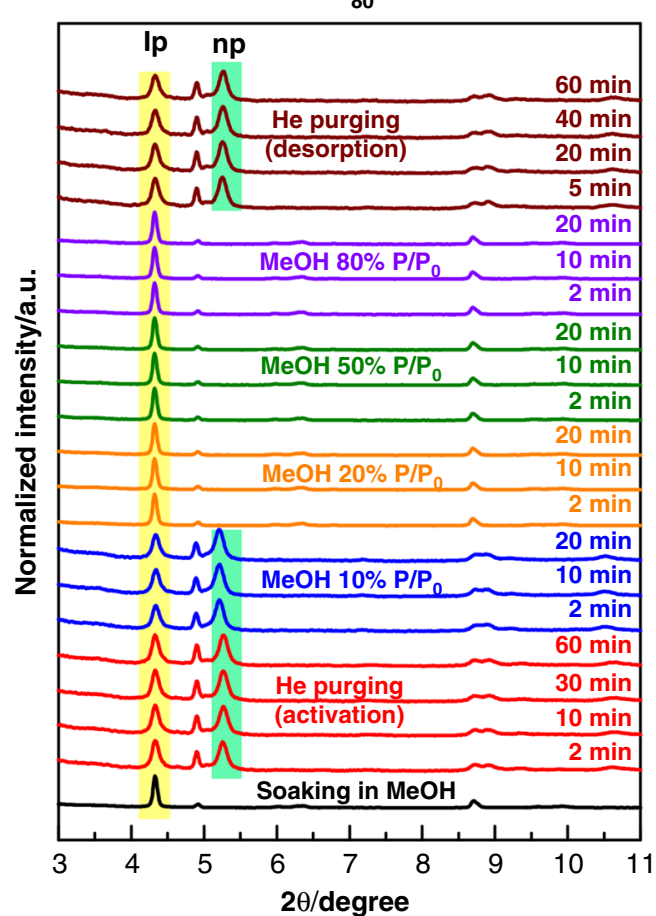

b

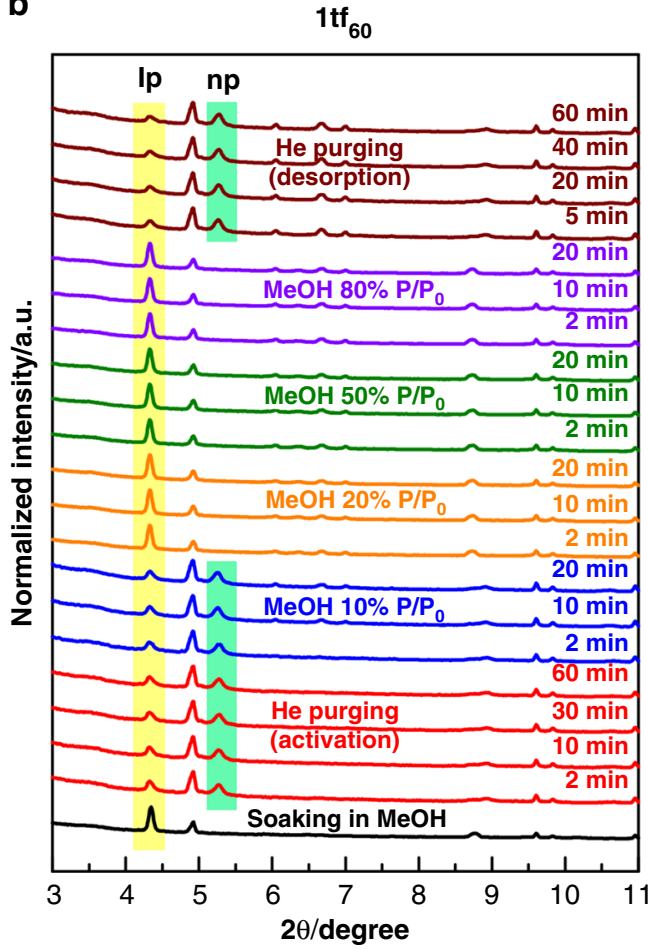

d

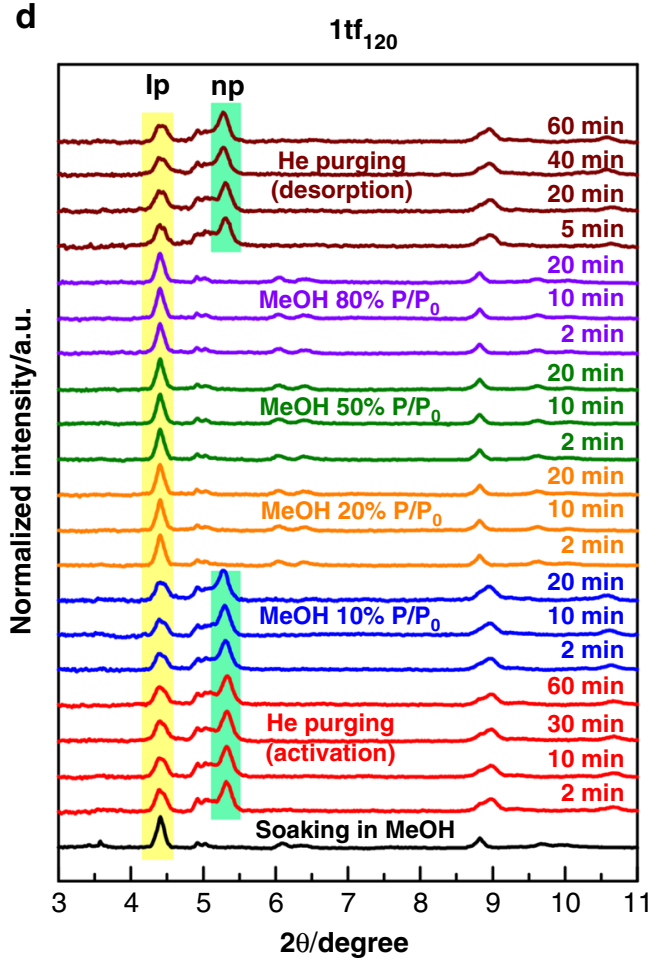

Fig. 4 Out-of-plane GIXRD profiles of the $\mathbf{1 t f}_{\mathbf{x}}$ materials during methanol sorption at $25^{\circ} \mathrm{C}$. Herein, a $\mathbf{1} \mathbf{t f} \mathbf{4 0}, \mathbf{b} \mathbf{1} \mathbf{t f}_{\mathbf{6 0}}, \mathbf{c} \mathbf{1 t f}_{\mathbf{8 0}}$ and $\mathbf{d} \mathbf{1 t f}_{\mathbf{1 2 0}}$ were fabricated on $\mathrm{COOH}$ functionalised QCM substrates, which show a preferred growth along the (110) plane ${ }^{37}$. Yellow and green highlighted bars represent the most intense diffraction peaks belonging to the $\mathbf{I p}$ and $\mathbf{n p}$ phase, respectively. Herein, $\mathbf{1 t f}_{\mathbf{x}}$ exhibit a significantly different structural flexibility upon methanol sorption than 1bulk. Moreover, a marked dependence of the structural flexibility on the total number of fabrication cycles is observed (see also Fig. 5)

flexibility, however there is no phase transition observed in the GIXRD. It strongly points towards that the breathing is more restricted in $\mathbf{1 t f}_{\mathbf{6 0}-\mathrm{Py}}$ than in $\mathbf{1 t f}_{\mathbf{6 0}}$. In $\mathbf{1 t f}_{\mathbf{6 0}-\mathrm{Py}}$, the whole $\mathrm{Cu}_{2}(\mathrm{DE}-$ bdc) $)_{2}$ grids are fixed parallel to the surface. The wine rack-like movement during the breathing transition would considerably affect the anchoring, disturbing the orientation of the SAM or putting a lot of stress on the interface. Hence we believe that the energy penalty caused at the interface cannot be compensated by the beneficial side chain-side chain and side chain-metal centre interactions of the np state. In other words, the flexibility is 
a

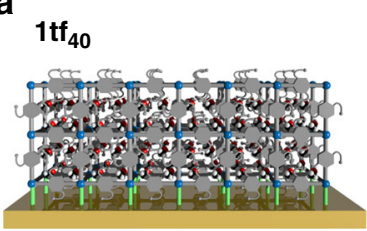

MeOH desorption (He purging)
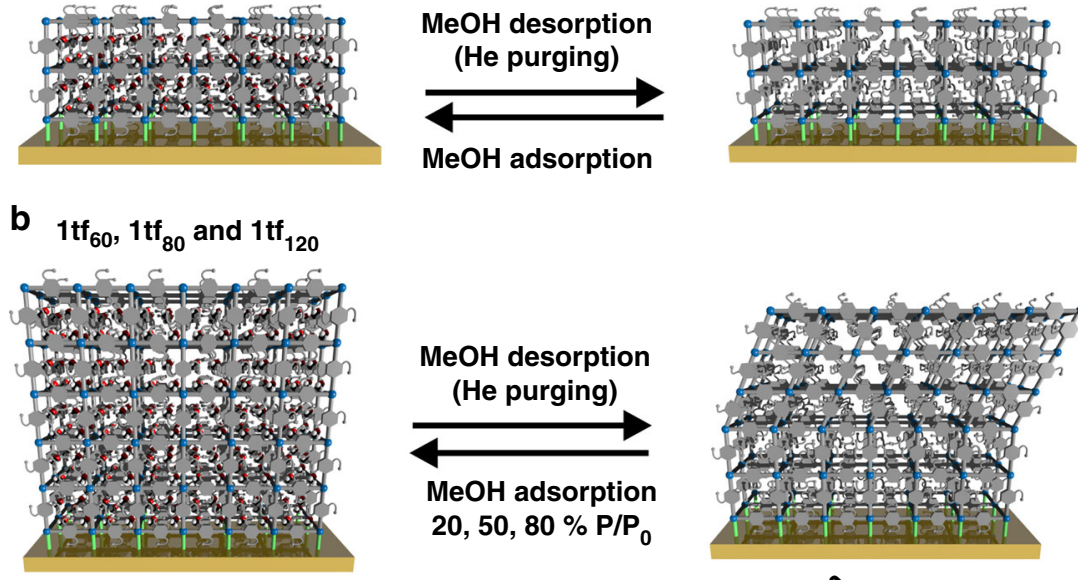

MeOH desorption (He purging)
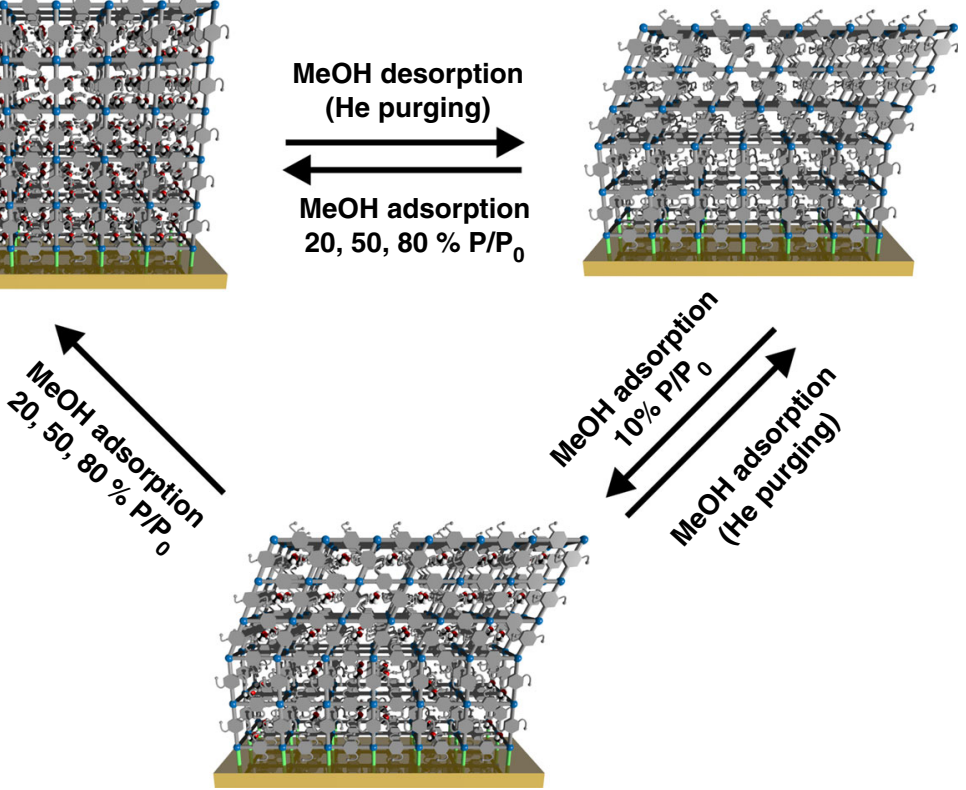

$\mathbf{5}$ Schematic representation of the structural response of $\mathbf{1 t f}_{\mathbf{x}}$ upon methanol sorption. $\mathbf{1 t f}_{\mathbf{x}}$ materials exhibit a unique structural transition depending on the total number of LPE fabrication cycles (i.e., the crystallite dimension and/or film thickness). a $\mathbf{1 t f}_{\mathbf{4 0}}$ remains in the $\mathbf{l p}$ form, regardless of the treatments,

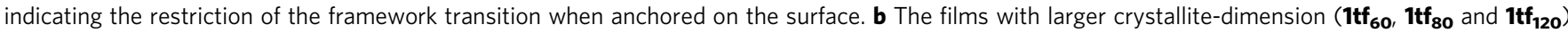
surprisingly reveal a unique structural flexibility. The crystallites domains close to the interface with the substrate surface remain in the Ip form, while the domains in sufficient distance to the surface can undergo a Ip-to-np breathing transition upon activation. Hence, both the $\mathbf{I p}$ and $\mathbf{n p}$ forms are presented at the activated stage. Adsorption of methanol vapour of at least $20 \% P / P_{0}$ is required for the reverse $\mathbf{n p}$-to-Ip structural transition to the solvated, Ip stage. Unlike the case of $\mathbf{1}$ bulk, $10 \% P / P_{0}$ of methanol vapour can be adsorbed in $\mathbf{1 t f}_{\mathbf{x}}$ but cannot induce the structural transition

inhibited if the growth direction of $\mathbf{1 t f}$ is changed. Notably, the guest-induced structural flexibility of $\mathbf{1 t f}$ depends not only on the crystallite dimension but also on the orientation of the crystallites anchored on the surface.

Furthermore, LPE is used for fabrication of $\mathbf{2} \mathbf{t f}_{\mathbf{6 0}}, \mathbf{2} \mathbf{t f}_{\mathbf{8 0}}$ and $\mathbf{2} \mathbf{t f}_{\mathbf{1 2 0}}$ on the $-\mathrm{COOH}$ functionalised QCM substrates. According to in-situ GIXRD profiles (Supplementary Figs. 23 and 24 ), the $\mathbf{2} \mathbf{t f}_{\mathbf{x}}$ materials also exhibit structural responsiveness upon methanol sorption in a similar manner to the $\mathbf{1 t f}_{\mathbf{x}}$ materials, which clearly indicates the guest-induced framework transition in related alkylether-functionalised MOF thin-films. In contrast, the pristine $\mathrm{Cu}_{2}(\mathrm{bdc})_{2}$ (dabco) (3) thin film fabricated by LPE for 60 cycles $\left(\mathbf{3 t f}_{\mathbf{6 0}}\right)$ does not show any structural reorganisation upon methanol sorption (Supplementary Fig. 25). This further emphasises that the alkylether pendent side chains are necessary for inducing structural flexibility upon polar-guest sorption into the $\mathrm{Cu}$-based layeredpillared MOFs, as well as their thin-films.

\section{Discussion}

We employed for the first time the stepwise LPE process to anchor a nanolayer of flexible layered-pillared MOF crystallites onto SAMfunctionalised QCM substrates. Variation of the SAM-functionalised surface and the total number of fabrication cycles controls the crystallite orientation and dimension (and/or thickness) of the thinfilms, as shown in previous works. Remarkably, the crystallite dimensions have a distinct effect on the structural dynamics. While the flexibility is inhibited for very thin films (40 deposition cycles), thicker films (60-120 deposition cycles) show a lp-to-np phase transition upon polar-guest desorption which is reversible upon readsorption. Additionally, the orientation of the film has a crucial impact on the transition, while samples grown along the (110) lattice plane show structural dynamics, these are inhibited for MOFs grown along the (001) lattice plane. We attribute the restriction of structural flexibility to the strong interaction between the crystallites and the SAM-functionalised substrate leading to a higher activation barrier for initiating the flexibility (such as in the low crystallitedimension $\mathbf{1} \mathbf{t f}_{\mathbf{4 0}}$ and the (001)-oriented $\left.\mathbf{1} \mathbf{t f}_{\mathbf{6 0}-\mathbf{P y}}\right)$. The breathing transformation occurs in the crystallite domains further away from the substrate interface, as the influence of the interface gets less pronounced. However, the effect of anchoring the flexible MOF crystallites onto the surface leads to increased threshold vapour pressures of methanol for initiating the np-to-lp transformation with respect to the bulk samples. In all, we provide a toolkit for the targeted preparation of MOF thin films with adjustable framework flexibility and demonstrate the fundamental understanding of breathing transitions within MOF thin films. The findings at hand are of relevant interest for specific applications such as MOF-thin film based sensors. A further extension towards well-controlled 


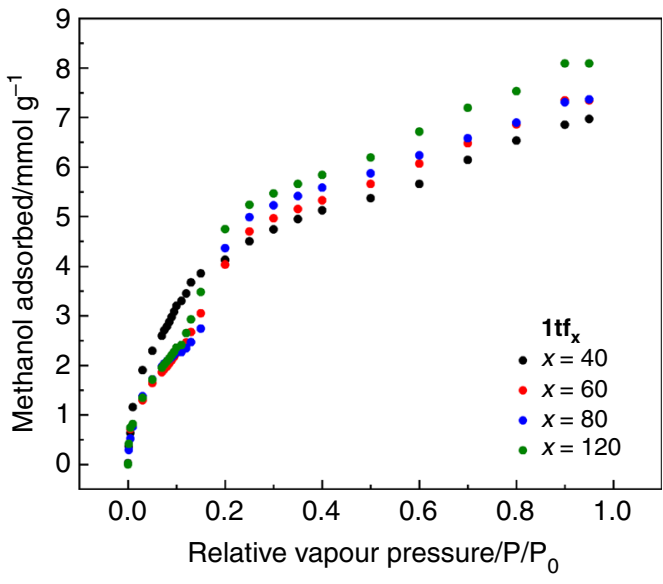

Fig. 6 Methanol adsorption isotherms of $\mathbf{1 t f}_{\mathbf{x}}$ measured at $25^{\circ} \mathrm{C}$ on an environmentally controlled QCM. $\mathbf{1 t f}_{\mathbf{4 0}}$ (black plots) exhibits a single-step methanol adsorption isotherm, whereas $\mathbf{1 t f}_{\mathbf{6 0}}$ (red plots), $\mathbf{1 t f}_{\mathbf{8 0}}$ (blue plots) and $\mathbf{1} \mathbf{t f}_{\mathbf{1 2 0}}$ (green plots) show two-step adsorption. The step in the adsorption profiles starting at $P / P_{O}=0.15$ indicates the threshold pressure that initiates the methanol-dependent np-to-Ip structural flexibility. Herein, it reveals the relation between the guest-induced structural transitions of $\mathbf{1} \mathbf{t} f_{\mathbf{x}}$ on the dimension of the MOF crystallites anchored to the surface (which is proportional to the number of LPE fabrication cycles, as well as the film thickness)

crystallite orientation ${ }^{54-56}$ and a combination of various responsivities by means of heterostructured architectures 57,58 is currently under development for the preparation of multiple-stimuliresponsive MOF-based devices.

\section{Methods \\ MOF thin-film fabrication. Au-coated QCM sensors (Q-Sense, Au electrode,} diameter $14 \mathrm{~mm}$, thickness $0.3 \mathrm{~mm}$ and fundamental frequency ca. $4.95 \mathrm{MHz}$ ) were used as substrates. Prior to the thin-film fabrication, the substrates were immersed in $20 \mu \mathrm{M}$ solution of 16-mercaptohexadecanoic acid (MHDA) in ethanol mixed with $5 \% \mathrm{v} / \mathrm{v}$ acetic acid for $24 \mathrm{~h}$ to generate a $-\mathrm{COOH}$ terminated surface. In order to generate a -pyridyl terminated surface, a $20 \mu \mathrm{M}$ solution of 4-(4-pyridyl)phenylmethylthiol (PMBT) in ethanol was used instead of the MHDA solution. 1tf and $2 \mathbf{t f f}_{\mathbf{x}}(x=40,60,80$ and 120 cycles) were fabricated by the LPE process at controlled temperatures of $40^{\circ} \mathrm{C}$ using an automated QCM instrument (Q-Sense E4 Auto) operated in the continuous flow mode with a constant flow rate of $100 \mu \mathrm{L}$ $\mathrm{min}^{-1}$. In each deposition cycle, the $-\mathrm{COOH}$ terminated QCM substrate was alternatingly exposed to the precursor solutions as follow: $\mathrm{Cu}(\mathrm{OAc})_{2} \cdot \mathrm{H}_{2} \mathrm{O}(0.5 \mathrm{mM}$ in ethanol) $10 \mathrm{~min}$, ethanol $5 \mathrm{~min}$, the mixed organic linkers $\left(\mathrm{H}_{2} \mathrm{DE}-\mathrm{bdc}+\right.$ dabco for $\mathbf{1} \mathbf{t f}_{\mathrm{x}}$ and $\mathrm{H}_{2} \mathrm{BME}-\mathrm{bdc}+$ dabco for $\mathbf{2} \mathbf{t f}_{\mathrm{x}}, 0.2 \mathrm{mM}$ in ethanol) $10 \mathrm{~min}$, and finally ethanol $5 \mathrm{~min}$. During the fabrication, the QCM frequency change was monitored in-situ. In addition, $\mathbf{1 t f}$ was also prepared on the - pyridyl terminated QCM by LPE for 60 cycles $\left(\mathbf{1 t f}_{\mathbf{6 0}-\mathbf{P y}}\right)$ in order to study the influence of crystallite orientation on the structural responsiveness of the MOF films. For comparison, the parent $\mathrm{Cu}_{2}(\mathrm{bdc})_{2}(\mathrm{dabco})$ thin film was also prepared by LPE for 60 cycles $\left(\mathbf{3 t f}_{\mathbf{6 0}}\right)$.

In-situ synchrotron $\mathbf{X}$-ray diffraction during methanol adsorption. Crystalline phase and structural flexibility during methanol sorption of the MOF powders and thin-films were identified by GIXRD (Beamline BL9 of the DELTA synchrotron radiation source, Dortmund, Germany. An X-Ray energy of $15.0 \mathrm{keV}$ corresponding to a wavelength of $0.827 \AA$ was used. The angle of incidence was $\left.0.6^{\circ}\right)^{59}$. In order to investigate the structural flexibility induced by polar-guest (i.e., methanol) sorption at ambient temperature $\left(25^{\circ} \mathrm{C}\right)$, the sample stage was equipped with a heatable graphite dome-type unit (DHS 1100, Anton Paar). This sample stage was further connected with the custom-built He gas flow system, which could semi-quantitatively control the dosing amount of methanol vapour into the sample chamber. The MOF sample was placed in the sample chamber and covered with the graphite dome. The temperature of the sample chamber was controlled at $25^{\circ} \mathrm{C}$ during the whole experiment. In-situ GIXRD patterns were recorded in different stages of the methanol sorption process as follows: (1) the as-synthesised, solvated stage (mimicking by drop methanol to the sample); (2) the activation (by purging the sample with He gas); (3) the methanol adsorption with 10, 20, 50 and $80 \% P / P_{0}$; and (4) the desorption (by purging with $\mathrm{He}$ gas flow). To control the $P / P_{0}$, the pressure-controlled valves at the pure $\mathrm{He}$ gas line and the one that passed through the methanol vaporiser were adjusted to change the mixing ratio, while keeping the total pressure at the end point behind the sample chamber constant (the schematic setup is shown in Supplementary Fig. 4).

Characterisation of MOF thin-films. Surface morphology and surface coverage were investigated by a field emission scanning electron microscopy (FE-SEM, ZEISS Gemini Sigma 300 VP). Cross-sectional SEM images were measured by a JEOL JSM-7500F operated in Gentle Beam mode without sputtering in order to determine the film thickness. Sorption properties of the MOF films were carried out on an environmental-controlled QCM (BEL-QCM-4 instrument, MicrotracBEL Corp.) at controlled temperature of $25^{\circ} \mathrm{C}$ using methanol as probe molecules. Prior to the sorption measurements, the films were activated in-situ within the BEL-QCM instrument by purging with a dry He gas with a flow rate of $100 \mathrm{sccm}$ for $2 \mathrm{~h}$ until the change of QCM frequency was stable within the range of $\pm 5 \mathrm{~Hz}$ in $20 \mathrm{~min}$. Then the mass of the MOF film was recorded by a conversion of the difference between the QCM frequency at the final activation and the fundamental frequency of the SAM-functionalised QCM substrates according to Sauerbrey's equation. After that, methanol sorption isotherms at $25^{\circ} \mathrm{C}$ were collected by varying $P / P_{0}$ of saturated methanol vapour in He gas flow from 0.0 to $95.0 \%$.

\section{Data availability}

The authors declare that the data supporting the findings of this study are available within the paper and its supplementary information file, which is available in the online version of the paper. Correspondence and requests should be addressed to R.A.F.

Received: 7 July 2018 Accepted: 22 December 2018

Published online: 21 January 2019

\section{References}

1. Hilf, R. J. C. \& Dutzler, R. Structure of a potentially open state of a protonactivated pentameric ligand-gated ion channel. Nature 457, 115-118 (2009).

2. Smith, D. E., Babcock, H. P. \& Chu, S. Single-polymer dynamics in steady shear flow. Science 283, 1724-1727 (1999).

3. Ichimura, K., Oh, S.-K. \& Nakagawa, M. Light-driven motion of liquids on a photoresponsive surface. Science 288, 1624-1626 (2000).

4. Berná, J. et al. Macroscopic transport by synthetic molecular machines. Nat. Mater. 4, 704-710 (2005).

5. Cohen Stuart, M. A. et al. Emerging applications of stimuli-responsive polymer materials. Nat. Mater. 9, 101-113 (2010).

6. Draper, J. W. A. Text-book on Chemistry. p. 55 (Harper \& Brothers Publisher, New York, 1861).

7. Hazen, R. M. \& Finger, L. W. Compressibility of zeolite 4A is dependent on the molecular size of the hydrostatic pressure medium. J. Appl. Phys. 56, 1838-1840 (1984).

8. Colligan, M. et al. Synchrotron X-ray powder diffraction and computational investigation of purely siliceous zeolite Y under pressure. J. Am. Chem. Soc. 126, 12015-12022 (2004)

9. Worthy, A. et al. Atomic resolution of structural changes in elastic crystals of copper(II) acetylacetonate. Nat. Chem. 10, 65-69 (2018).

10. Horike, S., Shimomura, S. \& Kitagawa, S. Soft porous crystals. Nat. Chem. 1 , 695-704 (2009).

11. Li, H., Eddaoudi, M. \& Yaghi, O. M. Design and synthesis of an exceptionally stable and highly porous metal-organic framework. Nature 402, 276-279 (1999).

12. Yaghi, O. M. et al. Reticular synthesis and the design of new materials. Nature 423, 705-714 (2004).

13. Kitagawa, S., Kitaura, R. \& Noro, S.-i Functional porous coordination polymers. Angew. Chem. Int. Ed. 43, 2334-2375 (2004).

14. Férey, G. Hybrid porous solids: past, present, future. Chem. Soc. Rev. 37, 191-214 (2008)

15. Murdock, C. R., Hughes, B. C., Lu, Z. \& Jenkins, D. M. Approaches for synthesizing breathing MOFs by exploiting dimensional rigidity. Coord. Chem. Rev. 258-259, 119-136 (2014).

16. Schneemann, A. et al. Flexible metal-organic frameworks. Chem. Soc. Rev. 43, 6062-6096 (2014)

17. Morris, R. E. \& Brammer, L. Coordination change, lability and hemilability in metal-organic frameworks. Chem. Soc. Rev. 46, 5444-5462 (2017).

18. Krause, $\mathrm{S}$. et al. A pressure-amplifying framework material with negative gas adsorption transitions. Nature 532, 348-352 (2016).

19. Serre, C. et al. Very large breathing effect in the first nanoporous chromium (III)-based solids: MIL-53 or CrIII $(\mathrm{OH}) \bullet\left\{\mathrm{O}_{2} \mathrm{C}^{\mathrm{II}} \mathrm{C}_{6} \mathrm{H}_{4}-\mathrm{CO}_{2}\right\} \bullet\left\{\mathrm{HO}_{2} \mathrm{C}-\mathrm{C}_{6} \mathrm{H}_{4-}\right.$ $\left.\mathrm{CO}_{2} \mathrm{H}\right\}_{\mathrm{x}} \bullet \mathrm{H}_{2} \mathrm{O}_{\mathrm{y}}$. J. Am. Chem. Soc. 124, 13519-13526 (2002).

20. Mellot-Draznieks, C., Serre, C., Surblé, S., Audebrand, N. \& Férey, G. Very large swelling in hybrid frameworks: a combined computational and powder diffraction study. J. Am. Chem. Soc. 127, 16273-16278 (2005). 
21. Seo, J., Matsuda, R., Sakamoto, H., Bonneau, C. \& Kitagawa, S. A pillared-layer coordination polymer with a rotatable pillar acting as a molecular gate for guest molecules. J. Am. Chem. Soc. 131, 12792-12800 (2009).

22. Maji, T. K., Matsuda, R. \& Kitagawa, S. A flexible interpenetrating coordination framework with a bimodal porous functionality. Nat. Mater. 6, 142-148 (2007).

23. Kitaura, R., Seki, K., Akiyama, G. \& Kitagawa, S. Porous coordination-polymer crystals with gated channels specific for supercritical gases. Angew. Chem. Int. Ed. 42, 428-431 (2003).

24. Yang, S. et al. A partially interpenetrated metal-organic framework for selective hysteretic sorption of carbon dioxide. Nat. Mater. 11, 710-716 (2012).

25. Chandler, B. D. et al. Mechanical gas capture and release in a network solid via multiple single-crystalline transformations. Nat. Mater. 7, 229-235 (2008).

26. Mason, J. A. et al. Methane storage in flexible metal-organic frameworks with intrinsic thermal management. Nature 527, 357-361 (2015).

27. Carrington, E. J. et al. Solvent-switchable continuous-breathing behavior in a diamondoid metal-organic framework and its influence on $\mathrm{CO}_{2}$ versus $\mathrm{CH}_{4}$ selectivity. Nat. Chem. 9, 882-889 (2017).

28. Horcajada, P. et al. Porous metal-organic-framework nanoscale carriers as a potential platform for drug delivery and imaging. Nat. Mater. 9, 172-178 (2010).

29. Yanai, N. et al. Gas detection by structural variations of fluorescent guest molecules in a flexible porous coordination polymer. Nat. Mater. 10, 787-793 (2011).

30. Loiseau, T. et al. A rationale for the large breathing of the porous aluminium terephthalate (MIL-53) upon hydration. Chem. Eur. J. 10, 1373-1382 (2004).

31. Millange, F. et al. Effect of the nature of the metal on the breathing steps in MOFs with dynamic frameworks. Chem. Commun. 39, 4732-4734 (2008).

32. Mowat, J. P. S. et al. A novel structural form of MIL-53 observed for the scandium analogue and its response to temperature variation and $\mathrm{CO}_{2}$ adsorption. Dalton Trans. 41, 3937-3941 (2012).

33. Devic, T. et al. Functionalization in flexible porous solids: effects on the pore opening and the host-guest interactions. J. Am. Chem. Soc. 132, 1127-1136 (2010).

34. Dybtsev, D. N., Chun, H. \& Kim, K. Rigid and flexible: a highly porous metal-organic framework with unusual guest-dependent dynamic behavior. Angew. Chem. Int. Ed. 43, 5033-5036 (2004)

35. Uemura, K., Yamasaki, Y., Komagawa, Y., Tanaka, K. \& Kita, H. Two-step adsorption/desorption on a jungle-gym-type porous coordination polymer. Angew. Chem. Int. Ed. 46, 6662-6665 (2007).

36. Wang, Z. \& Cohen, S. M. Modulating metal-organic frameworks to breathe: a postsynthetic covalent modification approach. J. Am. Chem. Soc. 131, 16675-16677 (2009).

37. Henke, S., Schneemann, A., Wütscher, A. \& Fischer, R. A. Directing the breathing behavior of pillared-layered metal-organic frameworks via a systematic library of functionalized linkers bearing flexible substituents. J. Am. Chem. Soc. 134, 9464-9474 (2012).

38. Henke, S., Schneemann, A. \& Fischer, R. A. Massive anisotropic thermal expansion and thermo-responsive breathing in metal-organic frameworks modulated by linker functionalization. Adv. Funct. Mater. 23, 5990-5996 (2013).

39. Schneemann, A. et al. Different breathing mechanisms in flexible pillaredlayered metal-organic frameworks: impact of the metal center. Chem. Mater. 30, 1667-1676 (2018).

40. Schwedler, I. et al. Mixed-linker solid solutions of functionalized pillared-layer MOFs-adjusting structural flexibility, gas sorption, and thermal responsiveness. Dalton Trans. 45, 4230-4241 (2016).

41. Furukawa, S., Reboul, J., Diring, S., Sumida, K. \& Susumu Kitagawa, S. Structuring of metal-organic frameworks at the mesoscopic/macroscopic scale. Chem. Soc. Rev. 43, 5700-5734 (2014).

42. Tanaka, D. et al. Rapid preparation of flexible porous coordination polymer nanocrystals with accelerated guest adsorption kinetics. Nat. Chem. 2, 410-416 (2010).

43. Sakata, Y. et al. Shape-memory nanopores induced in coordination frameworks by crystal downsizing. Science 339, 193-196 (2013).

44. Miura, H. et al. Tuning the gate-opening pressure and particle size distribution of the switchable metal-organic framework DUT- $8(\mathrm{Ni})$ by controlled nucleation in a micromixer. Dalton Trans. 46, 14002-14011 (2017).

45. Krause, S. et al. The effect of crystallite size on pressure amplification in switchable porous solids. Nat. Commun. 9, 1573 (2018).

46. Falcaro, P. et al. MOF positioning technology and device fabrication. Chem Soc. Rev. 43, 5513-5560 (2014)

47. Liu, J. \& Wöll, C. Surface-supported metal-organic framework thin films: fabrication methods, applications, and challenges. Chem. Soc. Rev. 46, 5730-5770 (2017).
48. Stassen, I. et al. An updated roadmap for the integration of metal-organic frameworks with electronic devices and chemical sensors. Chem. Soc. Rev. 46, 3185-3241 (2017)

49. Zacher, D. et al. Liquid-phase epitaxy of multicomponent layer-based porous coordination polymer thin films of $[\mathrm{M}(\mathrm{L})(\mathrm{P}) 0.5]$ type: importance of deposition sequence on the oriented growth. Chem. Eur. J. 17, 1448-1455 (2011).

50. Heinke, L., Tu, M., Wannapaiboon, S., Fischer, R. A. \& Wöll, C. Surfacemounted metal-organic frameworks for applications in sensing and separation. Microporous Mesoporous Mater. 216, 200-215 (2015).

51. Scherb, C., Koehn, R. \& Bein, T. Sorption behavior of an oriented surface grown MOF-film studied by in situ X-ray diffraction. J. Mater. Chem. 20, 3046-3051 (2010).

52. Haraguchi, T., Otsubo, K., Sakata, O., Fujiwara, A. \& Kitagawa, H. Guestinduced two-way structural transformation in a layered metal-organic framework thin film. J. Am. Chem. Soc. 138, 16787-16793 (2016).

53. Sakaida, S. et al. Crystalline coordination framework endowed with dynamic gate-opening behaviour by being downsized to a thin film. Nat. Chem. 8, 377-383 (2016).

54. Falcaro, P. et al. Centimetre-scale micropore alignment in oriented polycrystalline metal-organic framework films via heteroepitaxial growth. Nat. Mater. 16, 342-348 (2017).

55. Wannapaiboon, S. et al. Enhanced properties of metal-organic framework thin films fabricated via a coordination modulation-controlled layer-by-layer process. J. Mater. Chem. A 5, 13665-13673 (2017).

56. Virmani, E. et al. On-surface synthesis of highly oriented thin metal-organic framework films through vapor-assisted conversion. J. Am. Chem. Soc. 140, 4812-4819 (2018).

57. Wannapaiboon, S. et al. Hierarchical structuring of metal-organic framework thin-films on quartz crystal microbalance (QCM) substrates for selective adsorption applications. J. Mater. Chem. A 3, 23385-23394 (2015).

58. Wang, Z. et al. Directing the hetero-growth of lattice-mismatched surfacemounted metal-organic frameworks by functionalizing the interface. J. Mater. Chem. A 6, 21295-21303 (2018)

59. Krywka, C. et al. The small-angle and wide-angle X-Ray scattering set-up at beamline BL9 of DELTA. J. Synchrotron Radiat. 14, 244-251 (2007).

\section{Acknowledgements}

This work was initially supported by the Cluster of Excellence RESOLV (EXC 1069) and further by DFG FOR 2433 FLEXMOF funded by the German Research Foundation (DFG). DELTA is acknowledged for providing synchrotron radiation at beamline BL9 for grazing incidence X-ray diffraction measurements. S.W. acknowledges the Royal Thai Government under the Ministry of Science and Technology for a Ph.D. scholarship, the International Realisation Budget (IRB) of the Research School Plus, Ruhr-University Bochum (GSC 98) for international travel and research funding, and the Technical University of Munich for a Postdoctoral Fellowship. A.S. acknowledges the German Research Foundation for a Postdoctoral Fellowship. Katja Rodewald, Wacker-Chair of Macromolecular Chemistry, Technical University of Munich is acknowledged for measuring the cross-sectional SEM of the thin-film samples.

\section{Author contributions}

S.W. conceived, designed and performed the project under supervision of R.A.F; A.S synthesised the organic linkers and bulk powder samples; S.W., A.S., I.H., M.T., K.E. performed the in-situ GIXRD at DELTA synchrotron facility; S.W. and A.L.S. collected the adsorption data and SEM images; C.S. and M.P. helped designing the setup for in-situ GIXRD during adsorption and provided helpful discussion and support during the measurement at DELTA; A.S. and S.J.B. performed the Pawley refinements of GIXRD patterns of the powder samples; S.W., A.S., G.K. and R.A.F. co-wrote the paper

\section{Additional information}

Supplementary Information accompanies this paper at https://doi.org/10.1038/s41467 018-08285-5.

Competing interests: The authors declare no competing interests.

Reprints and permission information is available online at http://npg.nature.com/ reprintsandpermissions/

Journal peer review information: Nature Communications thanks the anonymous reviewers for their contribution to the peer review of this work. Peer reviewer reports are available.

Publisher's note: Springer Nature remains neutral with regard to jurisdictional claims in published maps and institutional affiliations. 
(c) (i) Open Access This article is licensed under a Creative Commons Attribution 4.0 International License, which permits use, sharing, adaptation, distribution and reproduction in any medium or format, as long as you give appropriate credit to the original author(s) and the source, provide a link to the Creative Commons license, and indicate if changes were made. The images or other third party material in this article are included in the article's Creative Commons license, unless indicated otherwise in a credit line to the material. If material is not included in the article's Creative Commons license and your intended use is not permitted by statutory regulation or exceeds the permitted use, you will need to obtain permission directly from the copyright holder. To view a copy of this license, visit http://creativecommons.org/ licenses/by/4.0/.

(C) The Author(s) 2019 